\title{
MECÁNicA CUÁNTICA Y LibRE AlbedRÍO: Cinco Cuestiones Fundamentales
}

\author{
JosÉ MANUEL MuÑOZ
}

\begin{abstract}
In this paper we critically analyze the situation of quantum mechanics in discussions on free will. It starts describing how the uncertainty principle and the measurement problem pose a challenge to determinism. Next, we present positions supporting and rejecting correlation between quantum phenomena and free will. Finally, we will place all these issues into the context of five key questions set out by Robert Kane: the Compatibility, Significance, Intelligibility, Existence and Determinist Questions.
\end{abstract}

Keywords: Free will; determinism; quantum mechanics; uncertainty principle; measurement problem.

\section{Introducción}

El propósito de este trabajo es efectuar un recorrido crítico por la situación en la que se encuentra la mecánica cuántica dentro del amplio y candente debate acerca de la relación entre determinismo y libre albedrío. Con el objetivo de que nuestro análisis sea lo más completo posible, apoyaremos este sobre la base proporcionada por cinco cuestiones que han sido planteadas por Robert Kane (1996, p.13; 2002, p.6) en alusión a la relación entre la libre voluntad y el determinismo, a saber: (A) Cuestión de Compatibilidad, (B) Cuestión de Significatividad, (C) Cuestión de Inteligibilidad, (D) Cuestión de Existencia, y (E) Cuestión Determinista. En concreto, las preguntas planteadas en las Cuestiones de Inteligibilidad y de Existencia acaparan actualmente una gran atención.

Es importante aclarar que, si bien las cinco cuestiones, dada la enorme envergadura de las preguntas que contienen, nos servirán para enmarcar el artículo, ello no significa que pretendamos analizar, defender o contrarrestar la postura personal de Kane acerca del libre albedrío. Por otro lado, omitiremos la presentación de los fundamentos del debate acerca de la relación entre libre albedrío y determinismo (e.g.: posibilidades alternativas, control último, compatibilismo, incompatibilismo, libertarismo). Algunos trabajos recientes que tratan dicha relación son: Fisher et al. (2007), Campbell (2011), Kane (2011), Griffith (2013).

En la sección 1 expondremos los aspectos esenciales de la mecánica cuántica, ${ }^{1}$ y lo haremos siguiendo el enfoque adoptado por la interpretación de Copenhague. Esta interpretación, la más comúnmente aceptada, posee un marcado tono indeterminista. 
Comenzaremos hablando sobre el descubrimiento de los cuantos, la dualidad ondapartícula y la no-localidad. Tras ello, trataremos sobre el principio de incertidumbre, el problema de la medida y el estudio probabilístico de los sistemas cuánticos. En la sección 2 presentaremos algunas interpretaciones alternativas que parecen dejar abierta la posibilidad de pensar la mecánica cuántica al modo determinista. En la sección 3 plantearemos posiciones y estudios sobre la posible correlación entre el libre albedrío y el indeterminismo cuántico. En la sección 4 discutiremos las consecuencias que los mecanismos y elementos teóricos previamente tratados traen consigo para las cinco cuestiones enunciadas por Kane. Concluiremos el trabajo con unas breves reflexiones.

\section{Aspectos Fundamentales de la Mecánica Cuántica. Interpretación de Copenhague}

\subsection{Descubrimiento de los Cuantos}

Hace más de un siglo, Max Planck (1900) descubrió que la energía de las ondas electromagnéticas no puede dividirse en fracciones, sino que se encuentra en paquetes, a los que denominó "cuantos", cuyo contenido es múltiplo de una unidad fundamental. Es más, el paquete de energía mínimo que puede presentar la onda, su unidad de energía, es directamente proporcional a su frecuencia (la cual, a su vez, es inversamente proporcional a la longitud de onda). Esta relación puede ser cuantificada mediante un factor llamado "constante de Planck" $(h)$, cumpliéndose la igualdad:

$$
E=h v,
$$

donde $E$ es la unidad de energía mínima de la onda electromagnética, $h$ es la constante de Planck y $v$ es la frecuencia de onda. El valor de $h$ es minúsculo (aproximadamente $6,63 \cdot 10^{-34} \mathrm{~J} \cdot \mathrm{s}$ ), de forma que los paquetes de energía tienen normalmente un tamaño pequeño. Los cambios de energía, igualmente, se producen en pequeños saltos, si bien estos son mayores conforme crece la frecuencia de onda (esto es, conforme disminuye la longitud de onda).

El planteamiento de Planck fue clave para las ideas de Niels Bohr (1913a, 1913b, 1913c) acerca de la estructura de los átomos, con los cuantos como elementos esenciales de esta. Sin embargo, Planck no encontró la forma de explicar por qué la energía se presenta en paquetes, algo que sí consiguió Albert Einstein investigando acerca del efecto fotoeléctrico. Este fenómeno consiste en la emisión de electrones por parte de algunos metales cuando son iluminados con radiación electromagnética. Cuando esta radiación choca con la superficie del metal, le transmite su energía, agitando algunos de los electrones de esta capa que están débilmente unidos a los átomos. Esta agitación provoca que los electrones puedan ser despedidos de la superficie metálica. 
El efecto fotoeléctrico posee ciertas características especiales. La intensidad de la radiación no influye de ningún modo en la velocidad de emisión de los electrones. Un aumento de la intensidad provoca que exista un mayor número de electrones emitidos, pero no consigue que aumente la velocidad a la que salen despedidos de la superficie del metal. Esta sí crece, por el contrario, cuando la frecuencia de la luz (radiación electromagnética) es mayor, es decir, cuando es menor su longitud de onda. También sucede lo contrario: un descenso en la frecuencia conlleva una disminución en la velocidad de emisión. Incluso llega un momento en que, cuando la frecuencia de la luz es lo suficientemente baja, los electrones dejan de ser emitidos, sin importar en ningún momento cuál sea la intensidad de la luz.

La aportación que Einstein realizó al estudio del efecto fotoeléctrico (Einstein, 1905) parte de la idea de integrar en la estructura de la luz la explicación de Planck acerca de los cuantos de energía de las ondas. Si dividimos la onda luminosa en paquetes, es decir, partículas de luz (a las que posteriormente se denominaría "fotones"), podemos encontrar una explicación al efecto fotoeléctrico. Cada fotón posee una energía que es proporcional a la frecuencia de onda, y esta ha de alcanzar un valor mínimo para que los electrones comiencen a ser emitidos desde la superficie metálica. En otras palabras, los fotones han de llevar asociada una energía mínima para que se produzca el efecto fotoeléctrico. Por otro lado, si aumentamos los electrones que contiene un rayo de luz, crece la intensidad de este, provocando que un número mayor de electrones sean agitados y emitidos, si bien su velocidad de emisión permanecerá constante mientras no varíe la frecuencia de la onda luminosa (y con ella la energía de los fotones). Einstein, de esta manera, dejó patente la eficacia de la explicación cuántica de Planck a la hora de describir la radiación electromagnética.

\subsection{Dualidad Onda-Partícula}

Isaac Newton afirmó que la luz está compuesta por partículas, pero su colega Thomas Young demostró, mediante el célebre experimento de la doble rendija, que el comportamiento de la luz obedece al de las ondas. Veamos la formulación moderna de este experimento, elaborada a partir de su formulación original. En una cámara oscura colocamos una fuente luminosa que dirigirá un haz de luz hacia una placa fotográfica situada en el lado opuesto. Entre ambas ubicamos una pared con dos rendijas verticales y paralelas entre sí. Si tapamos una de las dos rendijas, en la placa fotográfica quedará reflejada una única franja brillante como resultado del paso de la luz por la otra rendija, que permanece abierta. Pero si ambas rendijas están abiertas, la imagen reflejada en la placa no consistirá en dos franjas brillantes paralelas entre sí, como cabría esperar si la luz siguiera un comportamiento corpuscular. En su lugar, lo que se observa es un característico diagrama de interferencias, con alternancia de franjas oscuras y brillantes. Al interactuar la luz de ambas rendijas hay

Principia 19(1): 65-92 (2015). 
una interferencia de las ondas de modo que las crestas de algunos conjuntos de ondas se superponen con los valles de otros conjuntos, anulándose entre sí. Este tipo de interferencia produce las franjas oscuras. Sin embargo, otros conjuntos de ondas interfieren de modo que crestas y valles coinciden, aumentando la intensidad de la onda luminosa y produciendo las franjas brillantes.

El experimento de la doble rendija deja patente la naturaleza ondulatoria de la luz, pero las investigaciones de Einstein acerca del efecto fotoeléctrico demuestran igualmente su carácter corpuscular. Parece que los fotones, pese a ser partículas, se comportan de modo parecido a como lo hacen las ondas. De hecho, tal y como hemos podido ver, la energía de los fotones depende de la frecuencia, una característica que presentan las ondas. Basándose en todo esto, la mecánica cuántica proclama que la radiación electromagnética posee una dualidad onda-corpúsculo (dualidad ondapartícula). Según esta, las partículas pueden presentar un comportamiento ondulatorio y las ondas, a su vez, pueden comportarse tal y como lo hacen las partículas. Es más, Louis de Broglie (1925) propuso que la dualidad onda-partícula es una característica no sólo de la luz, sino de toda la materia. A la luz de nuestro conocimiento empírico actual, puede decirse que la propuesta era acertada; sin ir más lejos, los electrones manifiestan un patrón de interferencias similar al que presenta la luz.

\subsection{No-Localidad}

Cuando dos sistemas cuánticos interactúan, permanecen de algún modo correlacionados de manera que el estado de uno de los sistemas influye en el estado que poseerá el otro, y viceversa, sin importar que ambos se encuentren lejanos en el espaciotiempo. Esta misteriosa correlación fue rechazada por Einstein, Podolski y Rosen (1935), quienes formularon la llamada "paradoja EPR" con el fin de rebatir la nolocalidad cuántica. Décadas más tarde, no obstante, Aspect, Dalibard y Roger (1982) demostraron la no-localidad mediante un experimento de polarización ${ }^{2}$ con fotones. Observaron que si emitimos dos fotones simultáneamente y medimos la polarización de uno de ellos, existe una correlación con la polarización del otro de forma que el sistema compuesto por ambos es descrito mediante un único estado cuántico. Las polarizaciones de ambos fotones se influyen mutuamente aunque estén separados en el espacio. Pero, ¿qué es un estado cuántico? Lo veremos en el próximo apartado.

\subsection{Principio de Incertidumbre}

Si bien los cuantos, la dualidad onda-partícula y la no-localidad son elementos fundamentales de la mecánica cuántica, el punto de partida para comprender la relación entre esta y el determinismo reside en un descubrimiento efectuado por Werner Heisenberg (1927). Se trata del principio de incertidumbre, según el cual no podemos

Principia 19(1): 65-92 (2015). 
extraer simultáneamente, de modo preciso, el valor de dos observables de una partícula (tales como su posición y su momento lineal). La formulación matemática del principio, que adaptamos aquí a partir de la propuesta por Kennard (1927), es la siguiente:

$$
\Delta r \Delta p \geq h / 4 \pi
$$

donde $h$ es la constante de Planck, mientras que $\Delta r$ y $\Delta p$ corresponden a las desviaciones típicas (incertidumbres) en la posición y en el momento lineal de la partícula, respectivamente. Si queremos averiguar la posición y el momento lineal (o cantidad de movimiento) actuales de una partícula con el objetivo de predecir su posición y su cantidad de movimiento futuras, debemos iluminar dicha partícula. Seremos capaces de conocer la posición gracias a que algunas ondas electromagnéticas rebotan en ella y llegan a nuestra retina. Sin embargo, en esta determinación de la posición jugamos con un margen de error que es exactamente la distancia entre dos crestas consecutivas de la onda electromagnética, es decir, la longitud de onda. Por esta razón, para aumentar la precisión en el estudio de la posición será conveniente aplicar radiación con longitud de onda muy corta, o lo que es lo mismo, con muy alta frecuencia. Además, la mínima cantidad de luz que podemos aplicar durante la observación corresponde a un fotón (un cuanto de luz). El problema reside en que los fotones con corta longitud de onda albergan una gran cantidad de energía, de forma que al rebotar con la partícula en cuestión provocan inevitablemente una alteración en la cantidad de movimiento de esta. Si, por el contrario, utilizamos luz de alta longitud de onda, cuyos fotones poseen una menor cantidad de energía, la alteración sobre el momento lineal de la partícula observada será pequeña y podremos determinar con precisión dicho momento lineal. En este caso, el problema surgirá a la hora de averiguar la posición, puesto que el margen de error será mayor, dado que es igual a la longitud de onda, la cual será grande. En definitiva, cuanto mayor sea la precisión (menor la incertidumbre) con que hallemos la posición de una partícula, menor será la precisión (mayor la incertidumbre) con la que determinaremos su cantidad de movimiento; al revés, la relación funciona de la misma manera. Matemáticamente:

$$
\begin{aligned}
& \Delta p \geq h / 4 \pi \Delta r, \\
& \Delta r \geq h / 4 \pi \Delta p .
\end{aligned}
$$

Por si fuera poco, la incertidumbre se presenta, según Heisenberg, independientemente del tipo de partícula, del método utilizado y de las propiedades estudiadas. Por ejemplo, hay una relación inversa entre la precisión en la medida de la cantidad de energía de una partícula y el tiempo invertido en realizar dicha medición: no es posible conocer la energía de una partícula en un instante preciso. A raíz de todas estas observaciones, el estudio de las partículas en mecánica cuántica no se realiza 
a partir de la descripción separada de sus observables (como, por ejemplo, su posición y su momento lineal), sino de una combinación de ellas denominada "estado cuántico".

\subsection{Ondas y Probabilidad}

Max Born (1926) realizó una aportación a la teoría cuántica basada en estudiar las ondas electrónicas en términos probabilísticos: existen lugares de la onda donde es más probable encontrar el electrón que en otros. Esta explicación, ampliada a toda la materia, supone que esta, por su naturaleza ondulatoria, puede ser descrita mediante probabilidades. En los niveles macroscópicos esta descripción no tiene relevancia, puesto que la cualidad ondulatoria de la materia no se puede percibir, pero si nos centráramos en los niveles microscópicos nos encontraríamos con que la mejor manera de estudiar la posición de una partícula es asignarle probabilidades particulares de encontrarse en distintos lugares. No en vano, la repetición de un experimento bajo condiciones idénticas no garantiza idénticos resultados. Imaginemos que hacemos rebotar una onda electrónica contra una pared. Los electrones, tras encontrarse con el obstáculo, podrían encontrarse en lugares diversos para los cuales disponemos de probabilidades específicas. Si a continuación decidimos repetir el experimento en condiciones exactamente iguales a las del primer intento, posiblemente tendremos un resultado distinto en lo que se refiere a la posición de los electrones. Como observadores del experimento somos absolutamente incapaces de predecir la posición concreta en la que se encontrará un electrón. En su defecto, no tenemos otro remedio que asignar una probabilidad de que este, en efecto, se halle allí. Las predicciones pueden efectuarse gracias a la ecuación de Schrödinger, surgida a raíz de la investigación de este físico sobre la mecánica ondulatoria de las partículas (véase Schrödinger 1926a, 1926b, 1926c, 1926d). Puede expresarse de la siguiente forma:

$$
\hat{H} \Psi=i(h / 2 \pi)(d \Psi / d t),
$$

donde $\hat{H}$ es el operador hamiltoniano (referente a la energía total), $i$ es la unidad imaginaria $(i=\sqrt{-1})$ y $h$ es la constante de Planck. La ecuación de Schrödinger muestra cómo evoluciona la "función de onda" de un sistema cuántico $(\Psi)$, término acuñado para las ondas probabilísticas ya mencionadas. Ampliamente utilizada, esta ecuación ha dado lugar a predicciones muy eficaces en la investigación científica y tecnológica. Permite predecir la probabilidad de alcanzar diferentes futuros posibles (aunque, eso sí, no hay modo de saber cuál de ellos sucederá). Debemos aclarar, no obstante, que la probabilidad de que una partícula se halle en un lugar concreto en un momento determinado no viene dada por la función de onda, sino por el cuadrado de su valor absoluto, $|\Psi|^{2}$. También es importante tener presente que la ecuación

Principia 19(1): 65-92 (2015). 
de Schrödinger no constituye la única opción disponible a la hora de efectuar predicciones cuánticas. Existen otras vías, como la suma de historias posibles de Feynman, sobre la cual hablaremos en la sección 2.

¿Cómo debe ser interpretada la probabilidad cuántica? A este respecto, Heisenberg (1958, p.42) sostiene:

La probabilidad en matemáticas o en mecánica estadística significa una declaración sobre nuestro grado de conocimiento de la situación real. Al lanzar el dado no conocemos los sutiles detalles del movimiento de nuestras manos que determinan la caída del dado y, por tanto, decimos que la probabilidad de sacar un número en particular es exactamente de uno entre seis. La onda de probabilidad [...], sin embargo, significaba más que eso; significaba una tendencia hacia algo. Era una versión cuantitativa del viejo concepto de 'potencia' en la filosofía aristotélica [cursiva añadida]. Introducía algo situado en el medio entre la idea de un evento y el evento real, un extraño tipo de realidad física justo en el medio entre la posibilidad y la realidad. ${ }^{3}$

\subsection{Problema de la Medida}

Mientras que la física clásica supone que efectuar una medida saca a la luz propiedades intrínsecas del sistema, presentes en él antes de medirlo, la interpretación de Copenhague postula lo contrario: no tenemos razones para creer que las propiedades estaban presentes antes de que midiéramos el sistema. Por el contrario, este se encontrará en una superposición de estados que sólo se resolverá al medir, revelando un estado concreto. Este hecho provoca la aparición de lo que llamamos "problema de la medida" en mecánica cuántica. La razón de que sea un problema es que, en ausencia de medición, se considera que la función de onda que describe el sistema evoluciona de modo determinista, pero no puede explicar porqué la medida provoca una reducción desde una superposición de estados a uno solo en concreto. ${ }^{4}$

Una útil ilustración de este problema es una paradoja conocida como "el gato de Schrödinger". Se trata de un experimento imaginario concebido por el propio Schrödinger (1936). Pensemos en una caja en cuyo interior se aloja un gato. En ella encontramos también un recipiente que contiene una sustancia altamente venenosa, un detector de desintegración radiactiva y un martillo que interconecta ambos de manera tal que, cuando el detector refleja una desintegración radiactiva, el martillo rompe el recipiente y el veneno es liberado, matando al animal. Si no hay desintegración, no se libera veneno alguno y el gato continúa con vida. Cuando el experimento se va a llevar a cabo, se introduce en la caja una sustancia radiactiva que tiene un $50 \%$ de posibilidades de que uno de sus átomos se desintegre en el transcurso de una hora. Cuando la hora ha pasado, habrá la mitad de posibilidades de que el gato esté vivo y la mitad de que esté muerto, pero no podremos saber el desenlace si no abrimos la caja. Pues bien, según las leyes mecánico-cuánticas, la función de onda 
que describiría el estado del gato previamente a la apertura de la caja reflejaría la superposición de los estados "vivo" y "muerto": el gato no estaría concretamente vivo ni muerto, sino en los dos estados al mismo tiempo. Sólo podremos saber si el gato vive o ha muerto abriendo la caja, y al hacerlo modificaremos el sistema rompiendo la superposición de los dos estados, observando que, o bien el gato sigue con vida, o bien ha muerto. En ese momento de apertura de la caja, y sólo en ese momento, el gato estará vivo o muerto. Hasta ese instante estará vivo y muerto simultáneamente. ${ }^{5}$

El problema teórico suscitado por la paradoja recién expuesta y el problema de la medida dejan patente la dificultad de aceptar, sin más, una única interpretación de los fenómenos cuánticos (la de Copenhague en esta ocasión).

\subsection{Desafío al Determinismo}

Según van Inwagen (1983, p.65), el determinismo es la conjunción de dos tesis: (1) "Para cada instante de tiempo, existe una proposición que expresa el estado del mundo en ese instante", y (2) "Si $p$ y $q$ son proposiciones cualesquiera que expresan el estado del mundo en algunos instantes, entonces la conjunción de $p$ junto con las leyes de la naturaleza implica q". En la misma obra y página, el autor define el determinismo como "la tesis según la cual, dados el pasado y las leyes de la naturaleza, existe sólo un futuro posible". A la vista de estas descripciones, resulta fácil visualizar el impacto que el principio de incertidumbre y el problema de la medida poseen sobre el determinismo. ¿Cómo podemos confiar en las leyes de la naturaleza como actor fundamental para alcanzar un solo futuro posible en cada instante de tiempo si existen leyes físicas cuya esencia es la impredecibilidad? Una respuesta posible sería que no tenemos por qué aceptar la impredecibilidad cuántica, pues la mecánica cuántica podría ser una teoría incorrecta o, como creía Einstein, incompleta. Sin embargo, ha constituido un pilar para el desarrollo científico y tecnológico hasta nuestros días, ${ }^{6}$ motivo por el cual parece razonable tomarla muy en serio. Otra posible respuesta sería que no hay por qué pensar que la impredecibilidad refleje un aspecto esencial del universo, sino más bien nuestra incapacidad tecnológica y/o intelectual para estudiar los niveles más minúsculos del universo. En otras palabras, podríamos pensar que el indeterminismo cuántico no se presenta a nivel ontológico, sino sólo a nivel epistemológico. Esta posibilidad de elección entre dos interpretaciones distintas del indeterminismo cuántico ha sido destacada por Bishop (2002, p.117-8). La causalidad presente en la mecánica cuántica, dice Bishop, es una causalidad de tipo probabilista, y las probabilidades en las que se basa pueden tener dos naturalezas: epistemológica y ontológica. El primer caso abre la posibilidad de que la mecánica cuántica pueda ser de naturaleza determinista. En el segundo caso, el indeterminismo es una propiedad esencial de la mecánica cuántica, destruyendo la posibilidad del determinismo universal.

Principia 19(1): 65-92 (2015). 


\section{Interpretaciones Alternativas y Determinismo}

Si bien hasta este momento hemos considerado los fundamentos básicos de la mecánica cuántica a través de su interpretación más ampliamente aceptada (la de Copenhague), existen muchas otras, de entre las cuales expondremos tres que parecen abrir la puerta al determinismo cuántico: la suma de historias posibles de Feynman, la interpretación de los mundos múltiples y la interpretación de Bohm. ${ }^{7}$ Estas interpretaciones nos serán de utilidad para nuestro análisis de la Cuestión Determinista (sección 4).

Richard Feynman (1948) propuso un método denominado "suma de historias posibles", en el cual se considera que una partícula, en su viaje entre dos puntos cualesquiera, no toma una única trayectoria. En lugar de ello, recorre todos los caminos posibles entre ambos puntos. La probabilidad de que el fotón o cualquier otra partícula haga el recorrido entre dos puntos se obtiene sumando todas las trayectorias posibles entre ambos. Sin embargo, cuando tratamos con objetos macroscópicos, sucede que las trayectorias se anulan mutuamente exceptuando una sola, que es la que podemos percibir. Esta propuesta de Feynman guarda relación con la denominada "interpretación de los mundos múltiples", defendida en primer lugar por Everett (1957) y más tarde por otros (e.g.: Gribbin 1985, Deutsch 1998). En ella se afirma que, al efectuar una medición, el observador se relaciona con el sistema medido de manera que no tiene lugar realmente una reducción desde una superposición de estados a uno solo. Como indica Hodgson (2002, p.95-6), si seguimos la interpretación de los mundos múltiples el gato de Schrödinger sería observado tanto vivo como muerto. Las dos observaciones tendrían lugar, si bien una no sabría acerca de la otra.

En la interpretación de David Bohm (1952a, 1952b; Bohm y Hiley 1993) se sugiere la posibilidad de que existan variables ocultas influyendo sobre las probabilidades cuánticas y se niega la influencia del observador sobre los resultados. Las partículas poseen posiciones definidas, sin importar si se realiza una medición sobre ellas o no. Además, existe un campo llamado "campo cuántico", que afecta al movimiento de las partículas pero que es distinto de otros campos como el gravitatorio o el electromagnético. Esto se debe a que su fuerza no influye sobre su efecto (aunque sí lo hace su forma), es decir, al aumentar la distancia no disminuye el efecto del campo cuántico. Esta propiedad hace impredecibles las posiciones de las partículas, de manera que las probabilidades de la interpretación de Copenhague resultan eficaces a la hora de estudiarlas. El campo cuántico, además, provoca que dos partículas puedan estar correlacionadas pese a estar mutuamente distantes, dando cuenta así del fenómeno de la no-localidad. Ahora bien, no existe evidencia experimental a favor de la existencia del campo cuántico descrito por Bohm. En cualquier caso, su interpretación puede ser considerada como determinista porque supone que la conjunción de los eventos pasados y las leyes naturales conduce a un único futuro posible en cada instante de tiempo.

Principia 19(1): 65-92 (2015). 


\section{3. ¿Existe una Correlación entre el Indeterminismo Cuántico y el Libre Albedrío?}

A la vista de la posibilidad de que la mecánica cuántica sea indeterminista desde el punto de vista ontológico, cabe preguntarse si los fenómenos cuánticos, así interpretados, pueden constituir un correlato físico del libre albedrío concebido al modo de los libertaristas, para quienes este efectivamente existe pero no es compatible con el determinismo. Se trata de un debate controvertido, en el que han estado involucrados científicos y filósofos de diversa índole. En esta sección efectuaremos un recorrido general sobre este debate, lo que nos permitirá disponer de elementos de juicio esenciales para nuestro análisis en la sección 4.

El físico Roger Penrose ha sostenido que la mecánica cuántica podría hacer posible el libre albedrío. Considera (Penrose 1989, 1994) que la consciencia de los seres humanos es en gran parte no-algorítmica, no utilizando en muchas ocasiones mecanismos normativos ni algoritmos para su funcionamiento, y por tanto no puede ser reproducida computacionalmente. Piensa Penrose que las máquinas, que disponen de un número finito de enunciados y reglas, no podrían funcionar exactamente igual que una mente humana. ${ }^{8}$ En cuanto a la consciencia, sería un requisito para la capacidad de comprensión, y esta a su vez para la racionalidad. Pero, ¿qué tipo de fenómenos físicos podrían explicar una consciencia no-algorítmica? La respuesta se dirige hacia el proceso de reducción desde una superposición de estados cuánticos a uno solo, proceso que, para Penrose, no está bien explicado por la interpretación de Copenhague. Dicha interpretación, recordemos, trata de describir la reducción mediante una explicación basada en probabilidades. El autor opina, sin embargo, que este fenómeno podría ser descrito mediante una explicación no-algorítmica, tal y como la necesaria para explicar la racionalidad y la consciencia de la que esta depende. Sugiere, junto con Stuart Hameroff, la existencia de una "reducción objetiva orquestada" ("Orch OR"): un conjunto de reducciones objetivas, no-locales y sincronizadas, entre los microtúbulos de numerosas neuronas cerebrales (Hameroff y Penrose 1996). En su teoría, ${ }^{9}$

los estados cuánticos superpuestos surgen en las proteínas de las subunidades de los microtúbulos ('tubulinas'), permanecen coherentes, y reclutan más tubulinas superpuestas hasta que se alcanza un umbral de masa-tiempoenergía (relacionado con la gravedad cuántica). A esa altura, el auto-colapso, o reducción objetiva (OR), sucede abruptamente. Equiparamos la fase de pre-reducción, de superposición coherente ('computación cuántica'), con los procesos pre-conscientes, y cada OR instantánea (y no computable), o autocolapso, con un evento consciente discreto. Las secuencias de eventos $\mathbf{O R}$ dan lugar a un 'flujo' de consciencia. Las proteínas asociadas a microtúbulos pueden 'afinar' las oscilaciones cuánticas de los estados superpuestos cohe-

Principia 19(1): 65-92 (2015). 
rentes; la OR está por consiguiente auto-organizada, u 'orquestada' ('Orch OR'). Cada evento Orch OR selecciona (de manera no computable) estados de las subunidades de los microtúbulos que regulan funciones sinápticas/neurales utilizando la señalización clásica (ibid., p.36).

Se trata de un fenómeno de naturaleza no-algorítmica que, cree Penrose, podría constituir una base física no sólo para la consciencia sino también para el libre albedrío.

Henry Stapp (1993, 1998, 1999, 2007) cree que la interacción entre el observador y el mundo que lo rodea es fundamental para entender la consciencia y podría ser importante para comprender el libre albedrío. Según Stapp, las propiedades cuánticas de las partículas no existen de forma independiente a la medición. En este sentido, y al contrario que Penrose, adopta un enfoque cercano al de la interpretación de Copenhague, si bien con un cierto toque idealista: las propiedades cuánticas existen como estructuras que poseen la información más precisa que podemos obtener. ${ }^{10}$ Para Stapp, "la información es la moneda de la realidad, no la materia: el universo es una estructura informacional, no sustantiva” (Stapp 1999, p.149). La medición, sostiene, consiste en un intercambio de información, la cual llega a nuestro cerebro a través de los órganos sensoriales y es procesada por él. El ser humano es una conjunción de su cuerpo, su cerebro y su mente, de forma que posee un conjunto de eventos conscientes (su mente) organizados por su cuerpo y su cerebro. Tanto el observador (un ser humano) como el sistema que mide forman parte de una entidad informacional al completo, donde la reducción a un único estado cuántico del sistema medido se correlaciona con el estado cerebral del observador y con su elección acerca de qué preguntas formular al sistema. Existe una imagen física para cada evento consciente. Por lo tanto, la de Stapp "es una teoría de eventos, donde cada evento tiene un aspecto atencional y un aspecto intencional" (Stapp 1999, p.161). Además, el principio de incertidumbre de Heisenberg acerca de ciertas propiedades de las partículas (como posición, velocidad o energía) puede aplicarse a los iones calcio pre-sinápticos, haciendo impredecible la descarga de neurotransmisores. Esto hace que se cree una amplia gama de posibilidades alternativas, entre las cuales la selección se realizaría a través del proceso de reducción a un único estado cuántico. Stapp niega, eso sí, que nuestros pensamientos estén exentos de constricción alguna. Más bien lo que sucede es que eventos conscientes y sucesos cuánticos forman parte de una misma estructura en la que los primeros no son reducibles a los segundos.

El neurofisiólogo John Eccles ha defendido una tesis dualista de interacción entre mente y materia, centrándose especialmente en la liberación de moléculas neurotransmisoras en las sinapsis de las neuronas del neocórtex cerebral (Eccles 1986 y 1994, Beck y Eccles 1992). Las dendritas de dichas neuronas se agrupan en grandes racimos denominados "dendrones", y cada una de ellas termina en un botón sináptico (también llamado "terminal sináptico”). Cada botón contiene una enorme 
cantidad de vesículas alojando miles de neurotransmisores en su interior, los cuales pueden ser liberados a la hendidura sináptica cuando la señal eléctrica neuronal llega al terminal. La probabilidad de que esto suceda es del $25 \%$, y sería resultado del indeterminismo cuántico. Eccles sostiene que la mente es capaz de modificar esta probabilidad en los botones sinápticos de un dendrón al completo, afectando al funcionamiento de los mecanismos del cerebro. Con esta explicación, el indeterminismo cuántico podría generar posibilidades alternativas y el "yo" consciente, concebido como sustancia no-material, podría gobernar algunos procesos físicos del cerebro como los relativos al control de nuestras decisiones o a la creación de intenciones.

Mientras que Penrose, Stapp y Eccles apuestan por la correlación entre el indeterminismo cuántico y el libre albedrío, otros autores como Ted Honderich y Daniel Dennett creen que no existe dicha correlación. Honderich (1993, p.37) sostiene que los sucesos cuánticos ocurren por azar, y no se puede hacer responsable a nadie de decisiones cuya fuente sea de naturaleza azarosa. Para Dennett (1984, p.77), el indeterminismo a escala cuántica no conduce al indeterminismo a niveles macroscópicos como el de las acciones humanas. Para que esto fuera así, cree que debería existir algún mecanismo con efecto amplificador que lo hiciera posible, pero también que no se ha detectado ningún mecanismo de ese tipo. La generación de posibilidades alternativas, que podría deberse a sucesos azarosos de origen cuántico, puede explicarse igualmente mediante la alusión a procesos aparentemente azarosos, pero que en realidad no lo son, originados por los ordenadores (1984, p.120). Por último, aunque finalmente los sucesos cuánticos fueran causa de nuestra libertad mediante un proceso de amplificación de sus indeterminaciones, no habría manera de saber si una persona puede ser hecha responsable de un determinado acto, debido a la estructura altamente compleja de nuestro cerebro (1984, p.136). Dennett (1991) también ha sido crítico con el interaccionismo, defendido entre otros por Karl Popper y por el ya mencionado John Eccles. ${ }^{11}$ Para los interaccionistas, la materia y la mente son dos sustancias fundamentales que no son reducibles a otras y que se relacionan mutuamente. Sin embargo, esta tesis parece chocar con el principio de conservación de la energía. Mario Bunge (1980, p.17) dice al respecto: "Si la mente inmaterial pudiera mover la materia, entonces crearía energía; y si la materia actuara sobre la mente inmaterial, entonces la energía desaparecería. En cualquier caso la energía... no sería conservada".

Una vía de investigación que podría resultar interesante tanto para los partidarios como para los detractores del correlato entre el indeterminismo cuántico y el libre albedrío es la relativa a la búsqueda de posibles indeterminaciones cuánticas en los procesos neurobiológicos. Weber (2005) ofrece un exhaustivo repaso de los mecanismos susceptibles de presentar este tipo de indeterminaciones, y señala que los sucesos aleatorios pueden estar presentes en cualquiera de las estructuras y momentos presentes en la transmisión del potencial sináptico. Además, sostiene que los procesos de

Principia 19(1): 65-92 (2015). 
medición cuántica son intrínsecamente aleatorios, y aboga por la superveniencia de lo biológico a partir de lo físico, afirmando que la aleatoriedad a nivel macrofísico ha de estar asociada a una aleatoriedad microfísica (ibid., p.667-8). Como consecuencia de ello, los mecanismos neurobiológicos analizados por Weber como susceptibles de presentar indeterminaciones son de naturaleza molecular. En concreto, analiza las posibilidades de tres procesos: el transporte de neurotransmisores, la difusión molecular, y la apertura y el cierre de canales iónicos.

El transporte de neurotransmisores es llevado a cabo mediante vesículas en cuyo movimiento participa el citoesqueleto celular. Ciertos autores (Penrose 1994, Hameroff y Penrose 1996) han sugerido que los microtúbulos (componentes del citoesqueleto) pueden manifestar coherencia cuántica cuando se encuentran en un ambiente lo suficientemente aislado, provocando un proceso indeterminista de transporte de vesículas, y presentar el fenómeno (ya mencionado) de reducción objetiva orquestada u Orch OR. Sin embargo, Weber rebate estos argumentos afirmando que no existen hallazgos experimentales que apoyen la hipótesis de coherencia cuántica en los microtúbulos, ${ }^{12}$ y que estos actúan simplemente a modo de cables por los que las vesículas, con ayuda de proteínas motoras (las kinesinas y las dineínas), son conducidas hacia la hendidura sináptica, pero que no intervienen en la liberación de los neurotransmisores de las vesículas (Weber 2005, p.668). En cuanto al fenómeno de difusión molecular, es decir, el movimiento espontáneo de moléculas entre dos espacios con tendencia a igualar la concentración entre ambos, se sabe que tiene lugar en el momento en que los neurotransmisores atraviesan la hendidura sináptica. Weber aclara que el funcionamiento de este proceso se considera determinista actualmente (Weber 2005, p.668-9).

El mecanismo más prometedor para la manifestación de indeterminaciones cuánticas, piensa Weber, es el de apertura y cierre de canales iónicos. Es por esta razón que presenta una detallada serie de argumentos a favor y en contra de esta vía (Weber 2005, p.669-71). La posibilidad de que la apertura y el cierre de canales iónicos manifiesten indeterminaciones cuánticas, explica el autor, nace de estudios realizados mediante una técnica conocida como "patch-clamping", y según los cuales estos canales parecen exhibir un patrón estocástico. En concreto, y fijándonos exclusivamente en una molécula, el comportamiento de un canal es irregular. Si nuestro estudio se centra, en cambio, en observar si un canal se abrirá o se cerrará, veremos que ambas opciones gozan de una probabilidad concreta, sin importar que ese mismo canal estuviera previamente abierto o cerrado. Otros estudios han mostrado, además, que lo que sucede en un canal iónico puede ascender al nivel neuronal: se han observado potenciales de acción surgidos de modo espontáneo en cultivos celulares. Estos estudios corresponden a Johansson y Arhem (1994), así como a Chow y White (1996). Weber sugiere que "Esto suscita la posibilidad teórica de que un solo evento azaroso, quizás un evento cuántico, pueda tener alguna influencia en el comportamiento de 
un animal complejo" (Weber 2005, p.670). No obstante, ofrece también argumentos y referencias en contra de esta posibilidad (Liebovitch y Toth 1991, Cavalcanti y Fontanazzi 1999), según los cuales el funcionamiento de apertura y cierre de los canales iónicos puede ser igualmente descrito por modelos deterministas de carácter caótico cuyo desarrollo temporal es impredecible. Esto deja patente la dificultad que se presenta cuando se pretende demostrar que el indeterminismo cuántico se encuentra en la base de mecanismos neurobiológicos que son claves en el comportamiento de los seres humanos, y que por tanto son susceptibles de serlo en el ejercicio de su libertad. Así lo señala también Weber: "No es suficiente con mostrar que eventos particulares a nivel molecular podrían crear la diferencia entre distintos estados macroscópicos; los indeterministas deben demostrar asimismo que esos eventos moleculares son intrínsecamente estocásticos" (Weber 2005, p.671).

Una interesante relación física no mencionada hasta el momento es la que podría producirse entre los fenómenos de la mecánica cuántica y los descritos por la teoría del caos. Algunos trabajos han sugerido la posibilidad de un efecto amplificador del caos sobre los indeterminismos cuánticos (e.g., Hobbs 1991). Los fenómenos caóticos se caracterizan por el enorme peso que las condiciones iniciales tienen en la evolución de sus modelos. Un cambio minúsculo puede tener enormes consecuencias. Se trata, no obstante, de modelos cuyo desarrollo, si bien impredecible, es de naturaleza determinista. Por todo esto, se hace necesario considerar la posibilidad de que una fluctuación cuántica indeterminista, que por sí sola podría tener efectos despreciables en lo relativo al funcionamiento macroscópico del cerebro, pueda verse amplificada y tener consecuencias sobre el comportamiento y la volición debido a la intervención del caos. Sin embargo, esta posibilidad no está exenta de problemas, como el escaso consenso empírico acerca del funcionamiento caótico del cerebro, la existencia de interpretaciones deterministas de la mecánica cuántica (la de Bohm, por ejemplo) o la posibilidad de que el cerebro constriña la amplificación de los efectos cuánticos (Bishop 2002, p.119-20).

\section{Mecánica Cuántica y Libre Albedrío: Una Relación y Cinco Puntos de Vista}

Llegados a este punto, es momento de emplear todos los elementos y teorías vistos en un análisis que los enmarque en el debate general sobre el libre albedrío. Llevaremos a cabo esta tarea a partir de las cinco cuestiones de Kane acerca del libre albedrío: (A) Compatibilidad, (B) Significatividad, (C) Inteligibilidad, (D) Existencia, y (E) Determinismo.

Principia 19(1): 65-92 (2015). 


\subsection{Irrelevancia para la Compatibilidad y la Significatividad}

Los problemas de la compatibilidad y la significatividad del libre albedrío están planteados por Kane mediante las siguientes cuestiones:

[A] La Cuestión de Compatibilidad: ¿Es el libre albedrío compatible con el determinismo? (Kane 1996, p.13)

[B] La Cuestión de Significatividad: ¿Por qué queremos, o deberíamos querer, poseer un libre albedrío que es incompatible con el determinismo? ¿Es este un tipo de libertad "que valga la pena querer" (por utilizar la útil frase de Dennett)? Y si es así, ¿por qué? (Kane 1996, p.13)

Respecto a la Cuestión de Compatibilidad, pensamos que es poco lo que tiene que decir la mecánica cuántica por sí sola. No puede contribuir con datos experimentales que ayuden a esclarecer si el determinismo y el libre albedrío son compatibles, puesto que la libre voluntad, caso de existir, es susceptible de manifestarse a la escala macroscópica del cerebro (el único órgano capaz de alojar el "yo" volitivo), y la mecánica cuántica no analiza eventos cerebrales sino microfísicos. A esto hay que añadir el papel central que el indeterminismo juega en la teoría. Sea o no un indeterminismo de naturaleza ontológica, lo cierto es que una teoría indeterminista será incapaz de decir algo acerca de una hipotética compatibilidad del determinismo con la libertad.

En cuanto a la Cuestión de Significatividad, la mecánica cuántica no puede aportar nada. No nos ayuda a responder por qué los seres humanos deseamos o desearíamos tener una libertad incompatible con el determinismo. El debate sobre esta cuestión quizá pudiera ser enriquecido con aportes empíricos procedentes de la psicología y referentes a los motivos de un deseo como el que esta cuestión plantea. Una teoría puramente física, como es la mecánica cuántica, no puede decirnos nada sobre este asunto.

Aunque, en definitiva, la mecánica cuántica resulta irrelevante para las Cuestiones de Compatibilidad y Significatividad, comprobaremos a continuación que no sucede lo mismo con las tres cuestiones restantes: de Inteligibilidad, de Existencia y Determinista. El papel de la mecánica cuántica en el debate sobre determinismo y libre albedrío depende, por tanto, del enfoque específico que adoptemos en nuestro estudio.

\subsection{El Control Último como Reto para la Inteligibilidad}

La formulación planteada para la Cuestión de Inteligibilidad es la siguiente:

[C] La Cuestión de Inteligibilidad: ¿Podemos darle sentido a una libertad o libre albedrío que es incompatible con el determinismo? ¿Es di-

Principia 19(1): 65-92 (2015). 
cha libertad coherente o inteligible? ¿O es, como reivindican muchos críticos, esencialmente misteriosa y terminantemente oscura? (Kane 1996, p.13)

¿Es, pues, inteligible una libertad incompatibilista? Puesto que los libertaristas son los únicos incompatibilistas que creen que los humanos gozamos de libre voluntad, la pregunta podría ser formulada de esta manera: “¿Es inteligible un libre albedrío libertarista?” El libertarismo pregona que el determinismo excluye las posibilidades alternativas y el control último del agente, y que ambas condiciones son necesarias para el libre albedrío. Por lo tanto, una respuesta afirmativa a la Cuestión de Inteligibilidad que se base en la mecánica cuántica debería pasar por ofrecer una explicación que dé cuenta de cómo esta, suponiéndose indeterminista, hace posibles las posibilidades alternativas y el control último sobre nuestras decisiones y/o acciones. Es importante destacar que aquí lo importante no es la verdad de la explicación, sino solo si resulta inteligible (si podemos concebir su contenido y si es coherente).

Veamos de qué manera podría la mecánica cuántica posibilitar la existencia de posibilidades alternativas. Al tratarse de una teoría basada en probabilidades (según la interpretación de Copenhague), en la que un resultado individual es impredecible, resulta sencillo imaginar que el azar cuántico pueda ocasionar alteraciones indeterministas que generen diferentes posibilidades en distintas neuronas o grupos de neuronas, constituyendo una fuente de alternativas sobre las que se pueda escoger. No sabemos si esto sucede, pero es concebible. Encontramos más problemas cuando abordamos la condición de control último, sobre la que seguidamente me centraré. En la sección 3 hemos presentado tres importantes posturas (Penrose, Stapp y Eccles) a favor del correlato entre indeterminismo cuántico y libre albedrío, que podrían proporcionar una fuente de argumentos a los libertaristas para apoyar su visión. Analizaremos, una a una, dichas posturas, además de la posibilidad de que el caos constituya un sistema amplificador del indeterminismo cuántico.

Una libre voluntad libertarista que integre la Orch $O R$, descrita por Hammeroff y Penrose como un conjunto de reducciones objetivas, no-locales y sincronizadas entre los microtúbulos de las neuronas del cerebro, tendría posibilidades de sortear el obstáculo que supone la amenaza al control último. La coordinación entre reducciones a un único estado de un gran número de neuronas es un fenómeno no basado en propiedades de neuronas individuales, sino en sus relaciones. Y estas relaciones bien podrían ser un correlato de la actuación de un agente con un poder causal de tipo no-local. No se trataría de que cada neurona individual produjera un efecto cuántico decisivo en las decisiones y/o acciones del agente. Por el contrario, la Orch OR sería una manifestación del pensamiento consciente del agente, no su causa, lo que abriría la posibilidad de que parte de ese pensamiento consciente fuera una manifestación de su libre voluntad. Un libre albedrío que integrara esta descripción tendría natu-

Principia 19(1): 65-92 (2015). 
raleza indeterminista pero no vería amenazado su componente de control último; sería, por tanto, inteligible.

Stapp cree que la consciencia se sustenta en la existencia de una entidad informacional formada por un sistema cuántico y su observador, en la que la reducción a un único estado cuántico del primero se correlaciona con el estado cerebral del segundo. Considera que la clave es el intercambio de información, no la materia. Sin embargo, un libre albedrío libertarista que se sustente en esta explicación es difícilmente concebible. Tal y como Stapp afirma, los pensamientos no están libres de constricción alguna, pero a su vez no son reducibles a sucesos microfísicos sino que forman parte de una estructura informacional más amplia. Esto hace imposible situar el lugar donde podría situarse el control causal dentro del dúo formado por observador y sistema cuántico. No parece haber una direccionalidad causal, sino más bien un sistema de flujo de información en el que ninguna de las partes es causa de la otra. Dadas estas circunstancias, la hipótesis de Stapp no es prometedora como elemento de una explicación para una libre voluntad libertarista, pues no puede dar cuenta adecuadamente de su condición de control último.

La hipótesis de Eccles, centrada en la capacidad de la mente para modificar la probabilidad de liberación de neurotransmisores en los botones presinápticos de los dendrones, afectando al funcionamiento de los mecanismos del cerebro, podría ofrecernos la posibilidad de que el "yo" consciente controle ciertos procesos cerebrales como la formación de intenciones o la toma de decisiones. Sin embargo, un elemento nuclear de esta hipótesis es el convencimiento de que este "yo" consciente es una sustancia inmaterial que interacciona con la materia. Una tesis dualista de este tipo no ayuda a completar y hacer inteligible una explicación libertarista para el libre albedrío, sino que, por el contrario, añade nuevos conflictos, como, por ejemplo, la naturaleza de la conexión entre esa sustancia inmaterial y el sistema nervioso. Además, y en relación con el segundo principio de la termodinámica, no sabemos cómo podría evitar esta hipótesis el problema que supondría la creación de nueva energía en un sistema en el que una mente inmaterial tuviera un efecto causal sobre la materia, como bien apunta Bunge.

Dejando a un lado las propuestas concretas de los autores recién mencionados, vale la pena comentar la posibilidad de que el fenómeno del caos, de naturaleza impredecible pero determinista, tenga un efecto amplificador sobre la mecánica cuántica de forma tal que sus indeterminaciones pudieran ascender a niveles macroscópicos. Es concebible que indeterminaciones puntuales puedan trepar desde los niveles microfísicos de escala cuántica hasta los niveles macrofísicos del cerebro, incrementándose sus efectos. Esto podría eventualmente generar posibilidades alternativas. Sin embargo, no es inteligible la forma en que esta interacción cuántico-caótica podría posibilitar la condición de control último. ¿Cómo podría un agente poseer el control sobre sus decisiones si el origen de estas depende, en último término y ex- 
clusivamente, de eventos aleatorios a escala microfísica? Una teoría libertarista del libre albedrío, si pretende ser inteligible, no puede confiar en el efecto amplificador del caos sobre los eventos cuánticos como única explicación.

Como podemos ver, resulta altamente problemático explicar la condición de control último en un libre albedrío libertarista basándonos en la mecánica cuántica. La única hipótesis susceptible de ser integrada en una explicación libertarista, de forma que ésta resulte inteligible, es la ofrecida por Hammeroff y Penrose. Las propuestas de Stapp y Eccles tienen enormes dificultades si se pretenden utilizar como elementos de una teoría perteneciente al libertarismo. Por otro lado, cuando Honderich sostiene que no podemos hacer responsable a nadie de decisiones cuya fuente sea de naturaleza azarosa, como podría ser el caso de los sucesos cuánticos, es posible que piense en la dificultad para explicar el control último. No obstante, la mecánica cuántica podría ayudar a comprender el surgimiento de posibilidades alternativas y ligarse a explicaciones para el control último no basadas en ella. En este sentido, no hay motivo para rechazar que un agente pueda ser responsable de decisiones en cuyo proceso de formación interviene la mecánica cuántica.

\subsection{Indicios contra la Existencia}

La hipotética existencia de un libre albedrío libertarista queda planteada de esta forma:

[D] La Cuestión de Existencia: ¿Dicha libertad existe en realidad en el orden natural? Y si es así, ¿dónde? (Kane 1996, p.13)

La Cuestión de Inteligibilidad, planteada en el apartado anterior, apelaba a la coherencia de las explicaciones libertaristas, sin entrar a valorar la existencia o inexistencia de las libertades que proclaman. Esta tarea es solicitada en la Cuestión de Existencia, la cual podría quedar formulada de la siguiente manera si la contextualizamos en el tema del presente trabajo: "iExiste en el orden natural una libertad incompatible con el determinismo sustentada en la mecánica cuántica?". Hasta la fecha no se ha producido un hallazgo en sentido afirmativo, pero sí podemos analizar los indicios empíricos que existen al respecto.

Las hipótesis de Stapp y Eccles, si bien son susceptibles de ser contrastadas experimentalmente en alguno de sus elementos, como la probabilidad de descarga de neurotransmisores, son imposibles de comprobar en otros componentes que son claves para su articulación. En el caso de la estructura informacional de Stapp hay una reducción a un único estado cuántico del sistema medido correlacionada con el estado cerebral del observador y su elección acerca de qué preguntas formular al sistema. El problema de esta explicación es que no tenemos forma de distinguir si el estado cerebral del observador nace de la reducción a un único estado o si dicha reducción

Principia 19(1): 65-92 (2015). 
surge del estado cerebral del observador. En cuanto a la propuesta de Eccles, el problema radica en su carácter interaccionista. Si bien este autor ofrece una detallada descripción anatómica y funcional de su hipótesis, no hay evidencia empírica de la existencia de una mente inmaterial que tenga efecto sobre el cerebro material. Y es lógico que así sea, pues una sustancia inmaterial no puede ser detectada con técnicas e instrumentos materiales como los utilizados por los investigadores experimentales. Abogar por una mente no material es una postura que no puede ser corroborada por la ciencia actual. En definitiva, no hay pruebas empíricas que sustenten la posible existencia de un tipo de libertad incompatibilista basado en la hipótesis de Stapp o en la de Eccles.

La hipótesis de reducción objetiva orquestada, descrita por Hammeroff y Penrose, tiene la ventaja de estar en gran parte compuesta por elementos susceptibles de ser estudiados experimentalmente. Al situar el proceso Orch OR en los microtúbulos de las neuronas cerebrales, estos autores nos ofrecen, al contrario que los anteriormente mencionados, un lugar concreto donde buscar. Para apoyar la hipótesis Orch $O R$ necesitaríamos encontrar un conjunto de reducciones objetivas, no-locales y sincronizadas en los microtúbulos, que provocaría un transporte indeterminista de las vesículas sinápticas. Sin embargo, y como dijimos en la sección 3 , Weber señala que no existen actualmente hallazgos experimentales que apoyen la coherencia cuántica que necesitan los microtúbulos para que se produzca la reducción objetiva orquestada, pero que, en cambio, sí parece haber indicios que apuntan a que los microtúbulos actuarían meramente como cables por los que las vesículas que contienen moléculas de neurotransmisor se dirigirían hacia la hendidura sináptica. Es por ello que el modelo Orch $O R$, a día de hoy, no goza de pruebas concluyentes a su favor, de manera que no podemos afirmar que exista una libre voluntad libertarista que lo integre.

La posible amplificación de las indeterminaciones cuánticas debida a la actuación del caos también está sujeta a la Cuestión de Existencia. A este respecto, Bishop destaca que existe escaso consenso empírico sobre el posible funcionamiento caótico del cerebro, y que no debemos olvidar la posibilidad de que este constriña la amplificación. Dennett apunta hacia la necesidad de un mecanismo amplificador para que el indeterminismo a escala cuántica pueda conducir al indeterminismo al nivel macroscópico de las acciones humanas, pero destaca que no se ha detectado mecanismo alguno de esta clase. Si atendemos a afirmaciones como las de estos dos autores, no es posible sostener la existencia de una clase de libertad incompatibilista basada en la amplificación caótica de indeterminaciones cuánticas en nuestros cerebros.

A la vista de lo expuesto, no podemos ofrecer una respuesta afirmativa a la Cuestión de Existencia, puesto que las principales hipótesis que podrían ser utilizadas para ello son poco susceptibles a la comprobación empírica (Stapp, Eccles) o no gozan de apoyo experimental suficiente (Hammeroff y Penrose, efecto amplificador del caos). No obstante, cabría profundizar en el estudio de estas últimas por su contenido 
altamente comprobable desde el punto de vista empírico.

\subsection{Dudas sobre el Determinismo}

El problema de la verdad del determinismo como ley universal queda planteado del siguiente modo:

[E] La Cuestión Determinista: ¿Es el determinismo cierto? (Kane 2002, p.6)

Si obtuviéramos un "no" a esta cuestión basándonos en la mecánica cuántica, la solución sería definitiva: un solo caso de indeterminismo es suficiente para rechazar el determinismo como ley universal. Pero no sucede igual a la inversa: aunque concluyéramos que todos los fenómenos cuánticos son deterministas, no deberíamos olvidar la posibilidad de encontrar indeterminaciones en otros ámbitos. El objetivo en este apartado es discutir de qué modo puede ayudar la mecánica cuántica a responder al problema del determinismo.

Si entendemos el determinismo como la doctrina según la cual sólo puede existir un único futuro dados un pasado y unas leyes de la naturaleza concretas, entonces el principio de incertidumbre (incapacidad de averiguar simultáneamente el valor de dos observables de una partícula) y el problema de la medida (superposición de estados que sólo se resuelve cuando se realiza una medición) pueden suponer una seria amenaza bajo la interpretación de Copenhague. Los partidarios de dicha interpretación consideran que el principio de incertidumbre no refleja nuestra incapacidad para ahondar en los secretos de la materia, sino que es una propiedad intrínseca de la naturaleza. Creen, además, que la lectura correcta del problema de la medida consiste en considerar que la superposición de estados previa a la medición es real, lo que conduce a conclusiones, a nivel microfísico, similares a la conclusión de la paradoja del "gato de Schrödinger" (en ausencia de medición, el gato estaría vivo y muerto al mismo tiempo). Por otro lado, en la interpretación de Bohm las partículas poseen propiedades definidas independientemente de si son medidas o no, y la impredecibilidad acerca de estas propiedades nace de los efectos de un campo cuántico. Esta interpretación, al contrario que la anterior, es determinista desde el punto de vista ontológico, si bien acepta el indeterminismo epistemológico de los fenómenos cuánticos. En cuanto a la suma de historias posibles de Feynman (la probabilidad de que una partícula haga un recorrido entre dos puntos se averigua sumando todas las trayectorias posibles entre ambos) y la interpretación de los mundos múltiples (múltiples observaciones que tienen lugar simultáneamente y que no saben nada unas acerca de las otras), pueden parecer ontológicamente deterministas a simple vista (muchos autores así lo consideran), pues eliminan la impredecibilidad de los sucesos cuánticos al considerar que ocurre un enorme número de ellos al mismo tiempo. Sin

Principia 19(1): 65-92 (2015). 
embargo, esta simultaneidad contradice la existencia de un único futuro, necesaria para aceptar el determinismo definido por van Inwagen (1983, p.65) de la siguiente manera: "El determinismo, es, intuitivamente, la tesis según la cual, dados el pasado y las leyes de la naturaleza, existe un solo futuro posible". El determinismo (al menos entendido de esta manera) no es posible si varios futuros tienen lugar al mismo tiempo.

A nuestro modo de ver, no existen motivos suficientes para decantarnos por alguna de estas interpretaciones en cuanto a su determinismo o indeterminismo se refiere. En primer lugar, la interpretación de la suma de historias posibles se ha demostrado eficaz como instrumento a nivel teórico, pero no se han encontrado indicios experimentales a su favor. En segundo lugar, la interpretación de los mundos múltiples no puede ser comprobada experimentalmente (al menos actualmente), pues prevé que las distintas observaciones que tienen lugar al mismo tiempo no se conocen entre sí. No tenemos forma de saber si la observación real que experimentamos tiene lugar, o no, al mismo tiempo que otras observaciones paralelas. En tercer lugar, la interpretación de Bohm es ciertamente interesante como alternativa a la interpretación de Copenhague, y lo es por su coherencia y por su capacidad para explicar fenómenos también integrados por aquella, como la no-localidad y las predicciones basadas en probabilidades. Sin embargo, tal y como dijimos en la sección 2, no hay evidencias empíricas de la existencia del campo cuántico previsto por Bohm. En cuarto y último lugar, tampoco existe un motivo irrenunciable que nos impulse a pensar que el principio de incertidumbre integrado en la interpretación de Copenhague refleja un indeterminismo ontológico de la mecánica cuántica, por las razones que expondremos a continuación.

Tal y como apuntábamos en la sección 1, cuando queremos averiguar al mismo tiempo la posición y el momento lineal de una partícula nos encontramos con una seria dificultad. Uno de los modos que tenemos de intentarlo consiste en iluminar dicha partícula para saber dónde se encuentra. El problema surge porque si iluminamos con luz de corta longitud de onda, la partícula verá alterado su momento lineal al rebotar la luz en ella (aunque conoceremos su posición con precisión), y si iluminamos con luz de alta longitud de onda, tendremos un gran margen de error a la hora de averiguar su posición (aunque el momento lineal no se verá muy alterado y lo podremos conocer con precisión). Esta forma de estudiar una partícula refleja la ineficacia del método a la hora de analizar objetivamente el sistema en estudio, porque el método influye en el resultado. ¿Por qué debemos suponer que, previamente a una medición que se muestra ineficaz, el sistema cuántico no posee propiedades intrínsecas? De un modo similar, podríamos encontrarnos ante esta situación con otros tipos de métodos y de observables. La afirmación de que no tenemos razones para creer que las propiedades estaban presentes antes de realizar la medición es obviamente correcta: no podemos saber si algo está ahí hasta que lo percibimos. Pero tampoco deja de ser 
cierta la afirmación contraria: no hay razones para rechazar que las propiedades ya estaban ahí antes de que nosotros las midiéramos. ${ }^{13}$ Frente a esto podría esgrimirse el célebre Teorema de Bell (1964), según el cual no es posible la reproducción de las predicciones mecánico-cuánticas por medio de una teoría de variables ocultas de carácter local. Sin embargo, debe aclararse que este teorema nos obliga a rechazar: 1) el realismo (i.e., que las propiedades del sistema cuántico existen previamente a su medición), 2) el principio de localidad, o 3) ambos a la vez. Esto deja abierta la posibilidad de una teoría realista no-local, como la interpretación de Bohm. Ahora bien, las teorías de variables ocultas, y entre ellas la de Bohm, quedan seriamente dañadas a la luz del teorema Kochen-Specker (Kochen y Specker 1967). Este teorema demuestra que en un sistema cuántico no es posible atribuir valores a todas sus propiedades observables con precisión y simultaneidad. Una atribución precisa y simultánea resulta alcanzable cuando se limita a las propiedades observables relativas a un contexto de medición específico. A este respecto, Lombardi, Castagnino y Ardenghi (2009, p.9) dicen que "todo intento de adjudicar valores actuales precisos a todos los observables del sistema, de modo tal que las probabilidades puedan interpretarse por ignorancia respecto de un estado subyacente a la manera de la mecánica estadística clásica, conduce a contradicción". No obstante, y a pesar de su potencia, el teorema Kochen-Specker no ha podido ser comprobado empíricamente. Además, hay posibles escapatorias que, por el momento, impiden que se haya llevado a cabo un experimento que, de un modo definitivo, confirme el teorema de Bell (Cabello 2007, p.60). Por todo ello, pensamos que no puede afirmarse que alguna de las interpretaciones de la mecánica cuántica demuestre definitivamente, desde un punto de vista ontológico, que el indeterminismo cuántico sea cierto o que no lo sea. Consideramos, en sintonía con otros autores (e.g., Balaguer 2010, p.147), que el debate al respecto es inconcluyente. En este sentido, cabe destacar que durante los últimos años han aparecido trabajos relevantes con opiniones muy distintas respecto al supuesto indeterminismo cuántico. Tanto Gerard 't Hooft (2007) como otros autores (e.g.: Sadiq y Ghafoor 2010, Hofmann 2012, Brassard y Raymond-Robychaud 2013) han mantenido posturas deterministas. Por el contrario, Conway y Kochen (2006, 2009) han construido el "Teorema del Libre Albedrío", mediante el cual pretenden demostrar la indeterminación en los fenómenos cuánticos. Este teorema ha suscitado un encendido debate (véase Tumulka 2007, Goldstein et al. 2010).

Antes de finalizar con la discusión acerca de la Cuestión Determinista, vale la pena recordar unas observaciones acerca del indeterminismo cuántico en relación con la neurobiología. En la sección 3 expusimos, siguiendo las directrices utilizadas por Weber, tres procesos neurobiológicos moleculares susceptibles de presentar indeterminaciones cuánticas: el transporte de neurotransmisores, la difusión molecular, y la apertura y el cierre de canales iónicos. En primer lugar, el transporte de neurotransmisores necesita de una coherencia cuántica para la cual no pueden presentarse

Principia 19(1): 65-92 (2015). 
hallazgos experimentales a su favor. En segundo lugar, la difusión molecular es tratada actualmente como un proceso determinista. En tercer lugar, para la apertura y el cierre de canales iónicos existen estudios que muestran un patrón estocástico del proceso, pero también se han confeccionado modelos deterministas que pueden describirlo. Por lo tanto, podemos decir que el indeterminismo cuántico es incierto al nivel de los fenómenos moleculares de la neurobiología, si bien debería profundizarse en su posible manifestación en los procesos de apertura y cierre de canales iónicos.

En conclusión: ciñéndonos a la mecánica cuántica, ¿es el determinismo cierto? Teniendo en cuenta los datos actuales de los que disponemos, y a la vista de las argumentaciones aquí expuestas, todo lo que podemos responder a esta Cuestión Determinista es: "no lo sabemos". Se trata de una cuestión fundamental sobre la que la ciencia tiene mucho que decir y, por tanto, debe seguir indagando.

\section{Reflexiones Finales}

Para concluir, nos gustaría efectuar tres breves reflexiones. En primer lugar, es posible que las indeterminaciones cuánticas proporcionen una fuente para el surgimiento de posibilidades alternativas sobre las que un agente pueda escoger. Esta posibilidad, por sí sola, otorga relevancia a la mecánica cuántica en el debate sobre el libre albedrío, independientemente de la relación que exista entre el control último del agente y los fenómenos cuánticos. En segundo lugar, no sabemos con certeza si el indeterminismo cuántico tiene origen ontológico o epistemológico. Encontrar una respuesta concluyente a la Cuestión Determinista resulta fundamental para contextualizar las discusiones entre compatibilistas e incompatibilistas, y la mecánica cuántica todavía tiene mucho que decir sobre dicha cuestión. Y en tercer lugar, suponiendo un indeterminismo ontológico de la mecánica cuántica, los libertaristas podrían integrarlo en sus argumentos, aunque se encontrarían con el difícil obstáculo de tener que explicar el control último para un libre albedrío con origen intrínsecamente azaroso. La hipótesis de Hameroff y Penrose tendría posibilidades de ayudar a sortear dicho obstáculo, aunque a día de hoy no goza de apoyo empírico. Otras hipótesis aquí expuestas muestran demasiados inconvenientes como para ser de ayuda al libertarismo a día de hoy. En cualquier caso, cualquier respaldo cuántico a las hipótesis libertaristas debería encaminarse hacia un sentido descendente de la causalidad, es decir, del nivel mental al nivel físico. De esta manera, los fenómenos cuánticos constituirían un correlato de la libre voluntad del agente... pero no como su fuente sino como su consecuencia. Sólo así podría eludirse el llamado "argumento de Mind", ${ }^{14}$ según el cual el indeterminismo impide el control último del agente porque implica aleatoriedad y no es posible ejercer control sobre aquello que es aleatorio.

Principia 19(1): 65-92 (2015). 


\section{Agradecimientos}

Este trabajo es fruto de la investigación llevada a cabo por el autor durante su etapa doctoral en la UNED, concretamente en el departamento de Lógica, Historia y Filosofía de la Ciencia. El autor desea agradecer a las personas responsables de la valoración del texto su dedicación y sus valiosas sugerencias para mejorarlo.

\section{Referencias}

Aspect, A.; Dalibard, J.; Roger, G. 1982. Experimental Test of Bell's Inequalities Using TimeVarying Analyzers. Physical Review Letters 49: 1804-7.

Bacciagaluppi, G.; Valentini, A. 2009. Quantum Theory at the Crossroads: Reconsidering the 1927 Solvay Conference. Cambridge: Cambridge University Press.

Balaguer, M. 2010. Free Will as an Open Scientific Problem. Cambridge, Massachusetts: MIT Press.

Beck, F.; Eccles, J. C. 1992. Quantum Aspects of Brain Activity and the Role of Consciousness. Proceedings of the National Academy of Sciences of the USA 89: 11357-61.

Bell, J. S. 1964. On the Einstein Podolsky Rosen Paradox. Physics 1: 195-200.

Bishop, R. C. 2002. Chaos, Indeterminism, and Free Will. In: R. Kane (ed.) The Oxford Handbook of Free Will. Nueva York: Oxford University Press, p.111-24.

Bohm, D. 1952a. A Suggested Interpretation of the Quantum Theory in Terms of "Hidden" Variables I. Physical Review 85: 166-79.

- 1952b. A Suggested Interpretation of the Quantum Theory in Terms of "Hidden" Variables II. Physical Review 85: 180-93.

Bohm, D.; Hiley, B. J. 1993. The Undivided Universe: An Ontological Interpretation of Quantum Mechanics. Abingdon, Reino Unido: Routledge.

Bohr, N. 1913a. On the Constitution of Atoms and Molecules. Philosophical Magazine Series 6(26): 1-25.

- 1913b. On the Constitution of Atoms and Molecules. Part II.- Systems Containing Only a Single Nucleus. Philosophical Magazine Series 6(26): 476-502.

- 1913c. On the Constitution of Atoms and Molecules. Part III.- Systems Containing Several Nuclei. Philosophical Magazine Series 6(26): 857-75.

Born, M. 1926. Zür Quantenmechanik der Stossvorgänge. Zeitschrift Für Physik A: Hadrons and Nuclei 37: 863-7.

Brassard, G.; Raymond-Robichaud, P. 2013. Can Free Will Emerge from Determinism in Quantum Theory? In: A. Suarez, P. Adams (eds.) Is Science Compatible with Free Will?: Exploring Free Will and Consciousness in the Light of Quantum Physics and Neuroscience. Nueva York: Springer, p.41-61.

Bunge, M. 1980. The Mind-Body Problem: A Psychobiological Approach. Oxford: Pergamon Press.

Cabello, A. 2007. Reducción de la comunicación mediante entrelazamiento: del "descubrimiento más profundo de la ciencia" a la "pseudo-telepatía cuántica". Revista Española de Física 21: 58-60.

Campbell, J. K. 2011. Free Will. Cambridge, Reino Unido: Polity Press.

Principia 19(1): 65-92 (2015). 
Cavalcanti, S.; Fontanazzi, F. 1999. Deterministic Model of Ion Channel Flipping with Fractal Scaling of Kinetic Rates. Annals of Biomedical Engineering 27: 682-95.

Chow, C. C.; White, J. A. 1996. Spontaneous Action Potentials due to Channel Fluctuations. Biophysical Journal 71: 3013-21.

Conway, J.; Kochen, S. 2006. The Free Will Theorem. Foundations of Physics 36: 1441-73.

- 2009. The Strong Free Will Theorem. Notices of the American Mathematical Society 56: 226-32.

da Costa, N. C. A.; de Ronde, C. 2013. The Paraconsistent Logic of Quantum Superpositions. Foundations of Physics 43: 845-58.

de Broglie, L. 1925. Recherches sur la théorie des quanta. Annales De Physique 3: 22-128.

de Ronde, C. 2012. La noción de potencialidad ontológica en la interpretación modal de la mecánica cuántica. Scientiae Studia 10: 137-64.

Dennett, D. C. 1984. Elbow Room: The Varieties of Free Will Worth Wanting. Cambridge, Massachusetts: MIT Press.

- 1991. Consciousness Explained. Londres: Penguin.

Deutsch, D. 1998. The Fabric of Reality. Londres: Penguin.

Eccles, J. C. 1986. Do Mental Events Cause Neural Events Analogously to the Probability Fields of Quantum Mechanics? Proceedings of the Royal Society of London B 227: 411-28.

- 1994. How the Self Controls Its Brain. Berlín: Springer.

Einstein, A. 1905. Über einen die Erzeugung und Verwandlung des Lichtes betreffenden heuristischen Gesichtspunkt. Annalen Der Physik 322: 132-48.

Einstein, A.; Podolsky, B.; Rosen, N. 1935. Can Quantum-Mechanical Description of Physical Reality Be Considered Complete? Physical Review 47: 777-80.

Everett III, H. 1957. "Relative State" Formulation of Quantum Mechanics. Reviews of Modern Physics 29: 454-62.

Feynman, R. P. 1948. Space-Time Approach to Non-Relativistic Quantum Mechanics. Reviews of Modern Physics 20: 367-87.

Fischer, J. M.; Kane, R.; Pereboom, D.; Vargas, M. 2007. Four Views on Free Will. Malden, Massachusetts: Blackwell.

Goldstein, S.; Tausk, D. V.; Tumulka, R.; Zanghì, N. 2010. What Does the Free Will Theorem Actually Prove? Notices of the American Mathematical Society 57: 1451-3.

Gribbin, J. 1985. In Search of Schrodinger's Cat: Quantum Physics and Reality. Nueva York: Bantam.

Griffith, M. 2013. Free Will: The Basics. Abingdon, Reino Unido: Routledge.

Griffiths, D. J. 2014. Introduction to Quantum Mechanics (2nd ed.). Harlow, Reino Unido: Pearson.

Hameroff, S. R. 2012. How Quantum Brain Biology Can Rescue Conscious Free Will. Frontiers in Integrative Neuroscience 6: 93.

Hameroff, S. R.; Penrose, R. 1996. Conscious Events as Orchestrated Space-Time Selections. Journal of Consciousness Studies 3: 36-53.

Heisenberg, W. 1927. Über den anschaulichen Inhalt der quantentheoretischen Kinematik und Mechanik. Zeitschrift Für Physik A: Hadrons and Nuclei 43: 172-98.

-1958. Physics and Philosophy: The Revolution in Modern Science. Londres: George Allen \& Unwin Ltd.

Hobbs, J. 1991. Chaos and Indeterminism. Canadian Journal of Philosophy 21: 141-64.

Principia 19(1): 65-92 (2015). 
Hodgson, D. 2002. Quantum Physics, Consciousness, and Free Will. In: R. Kane (ed.) The Oxford Handbook of Free Will. Nueva York: Oxford University Press, p.85-110.

Hofmann, H. F. 2012. Complex Joint Probabilities as Expressions of Reversible Transformations in Quantum Mechanics. New Journal of Physics 14: 043031.

Honderich, T. 1993. How Free Are You?: The Determinism Problem. Oxford: Oxford University Press.

Johansson, S.; Arhem, P. 1994. Single-Channel Currents Trigger Action Potentials in Small Cultured Hippocampal Neurons. Proceedings of the National Academy of Sciences of the USA 91: 1761-5.

Kane, R. 1996. The Significance of Free Will. Nueva York: Oxford University Press.

- 2002. Introduction: The Contours of Contemporary Free Will Debates. In: R. Kane (ed.) The Oxford Handbook of Free Will. Nueva York: Oxford University Press, p.3-41.

- (ed.). 2011. The Oxford Handbook of Free Will (2nd ed.). Nueva York: Oxford University Press.

Kennard, E. H. 1927. Zur Quantenmechanik einfacher Bewegungstypen. Zeitschrift für Physik 44: 326-52.

Kochen, S.; Specker, E. P. 1967. The Problem of Hidden Variables in Quantum Mechanics. Journal of Mathematics and Mechanics 17: 59-87.

Liebovitch, L. S.; Toth, T. I. 1991. A Model of Ion Channel Kinetics Using Deterministic Chaotic Rather than Stochastic Processes. Journal of Theoretical Biology 148: 243-67.

Lombardi, O.; Castagnino, M.; Ardenghi, J. S. 2009. Mecánica cuántica: interpretación e invariancia. Theoria 64: 5-28.

Lucas, J. R. 1961. Minds, Machines and Gödel. Philosophy 36: 112-27.

Penrose, R. 1989. The Emperor's New Mind: Concerning Computers, Minds, and the Laws of Physics. Oxford: Oxford University Press.

-. 1994. Shadows of the Mind: A Search for the Missing Science of Consciousness. Oxford: Oxford University Press.

Planck, M. 1900. Zur Theorie des Gesetzes der Energieverteilung im Normalspectrum. Verhandlungen Der Deutschen Physikalischen Gesellschaft 2: 237-45.

Rae, A. I. M. 2005. Quantum Physics: A Beginner's Guide. Oxford: Oneworld.

Sadiq, M.; Ghafoor, F. 2010. An Alternative Realization of 't Hooft's Quantum Determinism. Physics Letters A 375: 11-3.

Schrödinger, E. 1926a. Quantisierung als Eigenwertproblem (Erste Mitteilung). Annalen Der Physik 384: 361-76.

- 1926b. Quantisierung als Eigenwertproblem (Zweite Mitteilung). Annalen Der Physik 384: 489-527.

-1926c. Quantisierung als Eigenwertproblem (Dritte Mitteilung). Annalen Der Physik 385: 437-90.

-1926d. Quantisierung als Eigenwertproblem (Vierte Mitteilung). Annalen Der Physik 386: 109-39.

- 1936. Die gegenwärtige Situation in der Quantenmechanik. Naturwissenschaften 23: 807-12.

Stapp, H. P. 1993. Mind, Matter, and Quantum Mechanics. Berlín: Springer.

- 1998. Pragmatic Approach to Consciousness. In: K.H. Pribram (ed.) Brain and Values:

Principia 19(1): 65-92 (2015). 
Is a Biological Science of Values Possible. Mahwah, Nueva Jersey: Lawrence Erlbaum, p.23748.

1999. Attention, Intention, and Will in Quantum Mechanics. In: B. Libet, A. Freeman, K. Sutherland (eds.) The Volitional Brain: Towards a Neuroscience of Free Will. Exeter, Reino Unido: Imprint Academic, p.143-64.

- 2007. Mindful Universe: Quantum Mechanics and the Participating Observer. Berlín: Springer.

Susskind, L.; Friedman, A. 2014. Quantum Mechanics: The Theoretical Minimum. Nueva York: Basic Books.

't Hooft, G. 2007. A Mathematical Theory for Deterministic Quantum Mechanics. Journal of Physics: Conference Series 67: 012015.

Tegmark, M. 2000. Importance of Quantum Decoherence in Brain Processes. Physical Review E 61: 4194-206.

Tumulka, R. 2007. Comment on "The Free Will Theorem". Foundations of Physics 37: 186-97. van Inwagen, P. 1983. An Essay on Free Will. Oxford: Clarendon Press.

Weber, M. 2005. Indeterminism in Neurobiology. Philosophy of Science 72: 663-74.

José MANUEL MuÑoz

UNED - Universidad Nacional de Educación a Distancia (España)

jomuor@gmail.com

\section{Notas}

${ }^{1}$ Si el lector está interesado en introducirse en la física cuántica con bastante mayor profundidad de la que podemos ofrecer en este artículo, algunas opciones son: Rae (2005), Griffiths (2014), Susskind y Friedman (2014).

${ }^{2}$ Este fenómeno consiste en la oscilación de las ondas electromagnéticas en un solo plano (el plano de polarización), en contraste con la oscilación en todas las direcciones con la que se propaga normalmente una onda.

${ }^{3}$ En los últimos tiempos, un autor que ha venido realizando una lectura aristotélica en la línea de la que hemos citado es Christian de Ronde (e.g., de Ronde 2012).

${ }^{4}$ En la celéberrima Conferencia Solvay de 1927, Paul Dirac defendió que dicha reducción corresponde a una elección tomada por el mundo natural (consúltese Bacciagaluppi y Valentini 2009, p.166).

${ }^{5}$ Según de Ronde (2012, p.156),

[un] indicador que expresa la distancia respecto de las superposiciones clásicas se encuentra dado por la constitución de la superposición cuántica a partir de términos contradictorios, por ejemplo, "gato vivo" y "gato muerto", "spin para arriba" y "spin para abajo". De este modo, si tomamos en cuenta la superposición cuántica más allá de su expresión formal, se nos presenta entonces como fundamental la consideración de la contradicción tanto en términos formales como conceptuales o interpretativos.

¿Cómo puede ser pensada la contradicción? Hoy contamos con las lógicas paraconsistentes, que ciertamente podrían ser consideradas con el objetivo de avanzar en el camino de un desarrollo de la estructura formal de la teoría.

Principia 19(1): 65-92 (2015). 
La lógica paraconsistente se caracteriza por su permisividad hacia las contradicciones, y precisamente de esta índole es el enfoque que el propio de Ronde y Newton da Costa han adoptado en relación a las superposiciones cuánticas (da Costa y de Ronde 2013). Sostienen los autores:

Nuestra propuesta se centra en la idea de que valdría la pena desarrollar una nueva interpretación de las superposiciones cuánticas que tenga en cuenta la contradicción "justo desde el principio". [Planteamos] una aproximación paraconsistente a las superposiciones cuánticas que muestra la posibilidad de tener en cuenta las contradicciones también desde una perspectiva formal. Sin embargo, debería quedar claro que no asumimos que la lógica paraconsistente sea la "lógica auténtica" que debería reemplazar a la lógica clásica [...]. Desde nuestra perspectiva sostenemos que los físicos deberían reconocer la posibilidad de utilizar nuevas formas de lógica-como la lógica paraconsistente-que podrían ayudarnos a comprender rasgos de diferentes dominios de la realidad [...]. No creemos que haya una "lógica auténtica", sino más bien que sistemas lógicos distintos pueden ser útiles para desarrollar y comprender aspectos complementarios de la realidad (ibid., p.855-6).

${ }^{6}$ El láser, los aceleradores de partículas, los superconductores de alta temperatura, la televisión en HD, las comunicaciones vía satélite ... son sólo algunos de los avances tecnológicos a los que la mecánica cuántica ha contribuido decisivamente.

${ }^{7}$ Otra interpretación considerada habitualmente como determinista es la interpretación de las muchas mentes, que es omitida aquí por estar estrechamente relacionada con la suma de historias posibles y los mundos múltiples. También es digna de mención la interpretación Madhyamika, que guarda similitud con la de Bohm pero se ciñe a lo fenoménico. Además, existen alternativas no deterministas (la mayoría indeterministas y otras agnósticas): la interpretación de von Neumann, las historias consistentes, la interpretación transaccional, las teorías de colapso objetivo, etc. No obstante, el contraste entre la interpretación indeterminista más ampliamente aceptada (la de Copenhague) y las principales alternativas deterministas nos parece suficiente para poder analizar la Cuestión Determinista (véase el apartado 4 de la sección 4).

${ }^{8}$ Penrose se inspira en una teoría presentada en su día por J.R. Lucas (1961).

${ }^{9}$ Puede leerse una descripción reciente de la teoría Orch OR en Hameroff (2012).

${ }^{10}$ Stapp no reconoce que su tesis sea propiamente idealista, sino que piensa que posee elementos tanto idealistas como materialistas (Stapp 1999, p.159).

${ }^{11}$ El interaccionismo defendido por Popper y por Eccles se enmarca dentro de la teoría de los tres mundos: el mundo 1 está formado por lo físico, el mundo 2 por lo mental y el mundo 3 por lo cultural. Esta teoría forma también parte del pensamiento de Penrose.

12 Por su parte, Tegmark (2000) ha sido crítico con la relación entre coherencia cuántica y consciencia.

${ }^{13}$ Las teorías de colapso objetivo, sin ir más lejos, sostienen que se da una destrucción de la superposición de estados cuánticos incluso en ausencia de medición.

${ }^{14}$ Este término fue empleado por primera vez por Peter van Inwagen (1983), aludiendo al empleo frecuente de esta clase de argumento en la revista Mind.

Principia 19(1): 65-92 (2015). 